\title{
Association of serum transaminases with short- and long-term outcomes in patients with ST-elevation myocardial infarction undergoing primary percutaneous coronary intervention
}

\author{
Ming Gao', Yi Cheng ${ }^{1,2,3}$, Yang Zheng ${ }^{1}$, Weihua Zhang ${ }^{1}$, Lin Wang ${ }^{1}$ and Ling Qin ${ }^{1 *}$
}

\begin{abstract}
Background: Alanine transaminase (ALT) and aspartate aminotransferase (AST) are referred to as liver transaminases. Although used routinely in clinical practice for decades, their role as predictors of mortality has not been examined until recently. We studied the predictive value of these serum transaminases in patients with ST-segment elevation myocardial infarction (STEMI) treated with primary percutaneous coronary intervention (PCI).
\end{abstract}

Methods: We analyzed records of 2417 consecutive STEMI patients with no preexisting liver disease who were treated with primary PCl at the Cardiovascular Center in the First Hospital of Jilin University. The outcomes measured were all-cause mortality at the first month and at 2 years. The relationship between the baseline serum transaminase levels and primary outcome was determined.

Results: We found a significant correlation between elevated liver transaminases and the Killip classification $(P<0.001$ for ALT; $P<0.001$ for AST), cardiac troponin I $(P=0.002$ for ALT; $P<0.001$ for AST), infarct-related coronary artery ( $P=0.036$ for $\mathrm{ALT} ; P=0.011$ for AST), and pre-thrombolysis-in-myocardial-infarction (pre-TIMI) flow $(P<0.001$ for ALT and AST). The serum level of ALT and AST were high along with the increasing of the grade of Killip classification. The primary infarct-related coronary artery in patients with ALT $\geq 95$ th percentage was left anterior descending artery (56\%), followed by right coronary artery (36\%). The OR for all-cause mortality at 2 years for participants with ALT $\geq 95$ th percentage was 5.370 (95\% Cl: 2.899-9.948), 7.034 (95\% Cl: 3.718-13.307) after adjustment for age and gender and 1. 051 (95\% Cl: 0.302-3.652) after adjustment for all covariables. The OR for all-cause mortality at 2 years for participants with AST $\geq 95$ th percentage was 5.370 (95\% Cl 2.899-9.948) and 5.699 (95\% Cl 3.030-10.718) after adjustment for age and gender and 1.796 (95\% Cl: 0.588-5.481) after adjustment for all covariables. ALT (HR 1.004, 95\% Cl 1.001-1.006, $P=0.010)$ and AST (HR 0.999, 95\% Cl 0.998-1.000, $P=0.030$ ) were associated with early all-cause mortality in patients with STEMI treated with PCI but not at 2 years post-procedure, unless for AST and ALT levels $\geq 95$ th percentage. Moreover, short- and long-term outcomes were significantly worse when both AST and ALT levels $\geq 95$ th percentage $(P<0.001)$. Conclusions: Serum transaminases $\geq 95$ th percentage were associated with a significantly increased incidence of short- and long-term all-cause mortality.

Trial registration: Registration number: ChiCTR-EPC-16008199, 31 March 2016.

Keywords: ST-elevation myocardial infarction, Serum transaminase, Primary percutaneous coronary intervention

\footnotetext{
* Correspondence: 1071927028@qq.com

${ }^{1}$ The Cardiovascular Center, the First Hospital of Jilin University, 71 Xinmin

Street, Changchun 130021, China

Full list of author information is available at the end of the article
} 


\section{Background}

Alanine transaminase (ALT) and aspartate aminotransferase (AST) are referred to as liver transaminases. Although their levels have been tested routinely in clinical practice for decades, their role as predictors of mortality has not been examined until recently. ALT is found predominantly in hepatocytes and is a widely used specific serum marker of liver disease. AST is mainly derived from the liver; although, a significant portion is derived from other tissues, such as heart, red blood cells, and muscle, which makes it an imperfect marker of liver function. Recent studies have demonstrated an increasing interest in investigating the role of liver transaminases in independently predicting cardiac-related morbidity and mortality [1-3].

Several prospective epidemiological studies have suggested that hepatic dysfunction is common in cardiac disease $[4,5]$. If no other causes of liver injury are identified, the elevations of liver aminotransferases are associated with a higher incidence of cardiac-related mortality [6]. However, the results from these studies have been inconsistent and have revealed geographical variations in the association between ALT and all-cause mortality [7-11]. Little is known about the association between hepatic dysfunction and mortality in patients with ST-segment elevation myocardial infarction (STEMI) treated with stents or angioplasty for coronary artery stenosis. The goal of this study was to evaluate the association between elevated liver transaminases and all-cause mortality in patients with STEMI undergoing primary percutaneous coronary intervention (PCI) at 1-month and 2-years post-procedure.

\section{Methods}

\section{Study population}

In this study, we examined digital data from the First Hospital of Jilin University, Cardiovascular Department, which is located in an agricultural province of Northeast China, populated by members of the Chinese Han ethnic group. This prospective observational cohort study aimed to investigate the association between ALT and AST levels and the incidence of all-cause mortality at 1 month and 2 years following treatment. From January 1, 2013 to December 31, 2014, 6017 patients were hospitalized with a diagnosis of acute myocardial infarction. Among those patients, 3692 had ST-segment elevation myocardial infarction. From those patients, 1056 were excluded who did not undergo angiography. Finally we included in this study all 2636 (71.3\%) consecutive STEMI patients who underwent PCI without thrombolysis or conservative therapy. From this group, we excluded 219 patients for the following reasons: insufficient AST or ALT data $(n=126)$, cirrhosis $(n=1)$, fatty liver $(n=64)$, and hepatitis $(n=8)$. In addition, we excluded 14 patients of another ethnic group. Finally, we excluded 16 patients because there was missing followup information. The final number of patients enrolled in this study was 2417. Their baseline demographic data, medical history, laboratory data, and clinical data during hospitalization were retrieved from the department's heart disease electronic database (Table 1). The mean time to follow up was 2 years (30-1165 days) in the included patients. Angiographic features, angiographic results, and treatment characteristics are provided in Table 2. The study protocol was approved by the ethics review board of the First Hospital of Jilin University (No.2016-263).

\section{Laboratory data}

Peripheral blood samples were obtained at the time of admission and the following were tested: creatinine, cardiac troponin I (Tn I), creatine kinase $\mathrm{MB}(\mathrm{CKMB}), \mathrm{K}^{+}, \mathrm{Na}^{+}$, and N-terminal pro-brain natriuretic peptide (NTproBNP). Total cholesterol (TC), triglyceride (TG), highdensity lipoprotein cholesterol (HDL-C), glucose, ALT, AST, alkaline phosphatase (ALP), -glutamyl transpeptidase (GGT), and low-density lipoprotein cholesterol (LDL-C) were measured after 12-hours fasting following admission. All blood samples obtained at the time of admission were analyzed in the certified laboratory department of First Hospital of Jilin University. ALT and AST were determined using ultraviolet-lactate dehydrogenase method and ultraviolet-malic acid dehydrogenase method according to the manufacturers' instructions (Kehua BioEngineering, China). According to local recommended guidelines, an abnormal ALT level was defined as a serum concentration $>50 \mathrm{U} / \mathrm{L}$ for men and $>40 \mathrm{U} / \mathrm{L}$ for women; an abnormal AST level was defined as a serum concentration $>40 \mathrm{U} / \mathrm{L}$ for men and $>35 \mathrm{U} / \mathrm{L}$ for women.

\section{Protocol and definition}

STEMI was diagnosed in accordance with the European Society of Cardiology/American College of Cardiology consensus document, using at least two separate measurements for the following parameters: a past history of chest pain, diagnostic electrocardiographic changes, and serial elevation of serum cardiac biomarkers [12]. The Killip classification is a system used in individuals with an acute myocardial infarction, taking physical examination and the development of heart failure to predict and stratify their risk [13]. It was determined as follows: Killip class I, no clinical signs of heart failure; Killip class II, rales or crackles in the lungs, an $S_{3}$, and elevated jugular venous pressure; Killip class III, frank acute pulmonary edema; and Killip class IV, cardiogenic shock or hypotension and evidence of peripheral vasoconstriction [13].

All patients enrolled were pre-medicated with either 1) $300 \mathrm{mg}$ aspirin or 2) $600 \mathrm{mg}$ clopidogrel orally and unfractionated heparin (1000 IU/L intravenous injection). 
Table 1 Baseline characteristics of participants $(n=2417)$

\begin{tabular}{|c|c|}
\hline Characteristic & $\mathrm{N}(\%)$ or mean $\pm \mathrm{SD}$ \\
\hline \multicolumn{2}{|l|}{ Demographic data } \\
\hline Age (year) & $59.5 \pm 11.1$ \\
\hline Male, n (\%) & $1748(72)$ \\
\hline Living in the city & $11,148(48)$ \\
\hline Hospital days & $7.2 \pm 3.8$ \\
\hline \multicolumn{2}{|l|}{ Medical history (n) } \\
\hline Dyslipidemia & $931(38)$ \\
\hline Diabetes mellitus & $545(23)$ \\
\hline Hypertension & $1088(45)$ \\
\hline Myocardial disease & $160(7)$ \\
\hline Previous myocardial infarction & $24(1)$ \\
\hline Arrhythmias & $492(20)$ \\
\hline Previous stroke & $75(3)$ \\
\hline Malignancies & $6(0.2)$ \\
\hline Peripheral vascular disease & $24(1)$ \\
\hline COPD & $30(1)$ \\
\hline \multicolumn{2}{|l|}{ Infarct location by ECG } \\
\hline Inferior & $1189(49)$ \\
\hline Anterior & $1172(48)$ \\
\hline Lateral & $56(2)$ \\
\hline \multicolumn{2}{|l|}{ Killip classification } \\
\hline Cardiogenic shock & $101(4)$ \\
\hline Noncardiogenic shock & $2316(96)$ \\
\hline \multicolumn{2}{|l|}{ Laboratory data } \\
\hline $\mathrm{K}^{+}(\mathrm{mmol} / \mathrm{L})$ & $3.9 \pm 0.5$ \\
\hline Creatinine (mmol/L) & $75.3 \pm 29.8$ \\
\hline $\mathrm{Na}^{+}(\mathrm{mmol} / \mathrm{L})$ & $139.4 \pm 4.1$ \\
\hline Total cholesterol (mmol/L) & $4.5 \pm 1.0$ \\
\hline $\mathrm{HDL}-\mathrm{C}(\mathrm{mmol} / \mathrm{L})$ & $1.1 \pm 0.3$ \\
\hline LDL-C (mmol/L) & $2.8 \pm 0.9$ \\
\hline Triglycerides (mmol/L) & $1.7 \pm 1.2$ \\
\hline Glucose (mmol/L) & $7.1 \pm 3.1$ \\
\hline ALT (unit/L) & $55.5 \pm 108.3$ \\
\hline AST (unit/L) & $165.3 \pm 283.1$ \\
\hline ALP (unit/L) & $72.4 \pm 24.4$ \\
\hline GGT (unit/L) & $47.4 \pm 69.4$ \\
\hline Cardiac troponin I (ng/mL) & $20.9 \pm 60.9$ \\
\hline Creatine kinase MB (ng/ml) & $38.3 \pm 75.9$ \\
\hline NT-proBNP (pg/mL) & $1829.9 \pm 6019.6$ \\
\hline
\end{tabular}

$S D$ standard deviation, COPD chronic obstructive pulmonary disease, ECG electrocardiographic, HDL-C high-density lipoprotein cholesterol, LDL-C low-density lipoprotein cholesterol, $A L T$ alanine aminotransferase, AST aspartate aminotransferase, ALP Alkaline phosphatase, GGT y-glutamyl transpeptidase, NT-proBNP N-terminal pro-brain natriuretic peptide
Table 2 Angiographic features, angioplasty results, and mortality of participants $(n=2417)$

\begin{tabular}{|c|c|}
\hline Characteristic/Outcome & N (\%) \\
\hline \multicolumn{2}{|l|}{ Infarct related artery } \\
\hline RCA & $1044(43)$ \\
\hline LAD & $1,172(48)$ \\
\hline LCX & $201(8)$ \\
\hline \multicolumn{2}{|l|}{ Pre-TMl flow } \\
\hline TIMI-0 flow & $475(20)$ \\
\hline$\geq \mathrm{TIMI}-1$ flow & $1,942(80)$ \\
\hline Multivessel disease ( $\geq 2$ vessels) & $1145(47)$ \\
\hline \multicolumn{2}{|l|}{ Method of reperfusion, n (\%) } \\
\hline Balloon & $282(12)$ \\
\hline Stent implantation & $2135(88)$ \\
\hline \multicolumn{2}{|l|}{ All-cause mortality } \\
\hline 1-month death & $59(2)$ \\
\hline 24-month death & $128(5)$ \\
\hline
\end{tabular}

Coronary angiography was performed through the femoral or radial artery using standard techniques to evaluate coronary artery lesions. Multivessel coronary artery disease was defined as significant stenosis of two or more major coronary arteries, stenosis of $\geq 50 \%$ of the diameter in the left main or proximal segment of the left anterior descending artery (LAD), and stenosis of $\geq 70 \%$ of the diameter in two of these three vessels: left coronary artery, right coronary artery (RCA), and left circumflex coronary artery (LCX). An artery was considered infarct-related if one of the following criteria was present: definite or suspected thrombus, a ruptured or ulcerated plaque, the presence of thrombolysis-in-myocardial-infarction (TIMI) flow grade $\leq 2$, or stenosis $\geq 70 \%$ of the diameter. The coronary artery flow was assessed using TIMI flow grades [14]. The strategy for multivessel arteries disease in our center was infarct-related artery only according to current guideline recommendations which encourage culprit-only PCI in patients with STEMI and multivessel disease (excluding cardiogenic shock) $[15,16]$.

\section{Follow-up}

To monitor for all-cause mortality following discharge, the clinical course of the patients was monitored by telephone until death or April 1, 2016, whichever occurred first. In the case of patients who were lost to follow-up, we reviewed all medical records and contacted the patients' families. The endpoint of the present study was defined in separate analyses of all-cause mortality at 1-month and at 2-years post-procedure. Two independent observers performed statistical analysis. 


\section{Statistical analysis}

After testing for normality by the Kolmogorov-Smirnov test, the data were presented as mean \pm standard deviation (SD) or median [interquartile range]. Separate analyses were conducted for ALT and AST. Serum ALT and AST levels were categorized into percentiles separately ( $<25$ th, 25th to $<50$ th, 50th to $<75$ th, 75 th to $<95$ th, and $\geq 95$ th percentages). Liver enzyme levels $<25$ th percentage were used as a reference. Logistic regression was performed to evaluate the power of percentiles for each liver enzyme with all-cause mortality. AUC curves were constructed to further illuminate the best cut-off values for ALT and AST for predicting the all-cause mortality. Multiple linear regression was used in order to test the association of the liver enzymes and other previously identified prognostic factors in STEMI with the measured outcomes. Survival curves were generated by the KaplanMeier method, and survival among groups was compared using the Log Rank test. To evaluate the independent contribution of the baseline clinical characteristics, medical history, and laboratory data on the occurrence of major events, two consecutive models of Cox multiple regression analysis were performed with forward stepwise selection to determine predictors for all-cause mortality at 1 month and 2 years separately. In the first model, the baseline clinical characteristics and laboratory data were included. Only those clinical and laboratory variables found to be independent in the first model were included in the second model. For all analyses, a two-sided $P<0.05$ was considered statistically significant. All analyses were conducted using Stata software, version 12 (Stata Corp., College Station, TX).

\section{Results}

\section{Baseline characteristics}

The baseline data of the participants included demographic characteristics, medical history, laboratory data, culprit vessel, and PCI procedure. The median age of the 2417 patients was 60 years [interquartile range: 52-67] with $72 \%$ male patients. Using the definitions of abnormal liver enzyme levels defined previously, 38.9\% of the study population had elevated ALT and $71.9 \%$ of the study population had elevated AST (Table 1). During the median 2 years (ranging from 30 to 1165 days, median 775 days) of follow up, the cumulative data for all-cause mortality was $2 \%$ (59 deaths) at the first month and 5\% (122 deaths) at 2 years (Table 2).

\section{Risk factors and elevated liver enzymes}

We found some differences in the associations between known risk factors and elevated liver enzymes between ALT and AST. Variables found to be independent in the first regression model and further analyzed included age, gender, living in rural regions, hospital days, medical history, Killip classification, infarct-related coronary artery, Tn I, pre-TIMI, and multivessel disease. In multivariate regression analysis, elevated ALT and AST were both related to Killip classification $(P<0.001$ for ALT; $P<0.001$ for AST), Tn I $(P=0.002$ for ALT; $P<0.001$ for AST), infarct-related coronary artery $(P=0.036$ for ALT; $P=0.011$ for AST), and pre-TIMI flow $(P<0.001$ for ALT and AST). ALT was also related to age $(P=0.002)$ and dyslipidemia $(P=0.048)$ (Table 3$)$. The serum level of ALT and AST were high along with the increasing of the grade of Killip classification (Fig. 1). The primary infarct-related coronary artery in patients with ALT $\geq 95$ th percentage was LAD (56\%), followed by RCA (36\%).

At cox proportional hazards regression, ALT (hazard ratio [HR] 1.004, 95\% confidence interval [CI] 1.001-1.007, $P=0.010)$ and AST (HR 0.999, 95\% CI 0.098-1.000, $P=0.030)$ were associated with of all-cause mortality at 1 month but not at 2 years after adjustment for potential confounders (Additional file 1: Table S1 and Table S2).

\section{Transaminases stratification and clinical outcome}

Values for the percentages of ALT and AST in males and females in the study population are shown (Additional file 1: Table S3). The odds ratio (OR) for all-cause mortality at 2 years for participants with ALT $\geq 95$ th percentage was 5.370 (95\% CI: 2.899-9.948), 7.034 (95\% CI: 3.718-13.307) after adjustment for age and gender and 1.051 (95\% CI: 0.302-3.652) after adjustment for all covariables. The OR for all-cause mortality at 2 years for participants with AST $\geq 95$ th percentage was 5.370 (95\% CI 2.899-9.948), 5.699 (95\% CI 3.030-10.718) after adjustment for age and gender and 1.796 (95\% CI: 0.588-5.481) after adjustment for all covariables (Table 4). For predicting outcomes, we constructed the receiver operating characteristic area under the curve (ROC-AUC) to value the best cut-off of ALT and AST. Using a cut-off level of $63.65 \mathrm{IU} / \mathrm{L}$, ALT predicted 1 month mortality with a sensitivity of $75.19 \%$ and specificity of $50.85 \%$ (ROC-AUC: $0.635,95 \% \mathrm{CI}: 0.552$ to $0.719, P<0.001$ ) and 2 year mortality with a sensitivity of $75.56 \%$ and specificity of $44.26 \%$ (ROC-AUC: 0.604 , 95\% CI: 0.546 to $0.662, P<0.001)$. Using a cut-off level of $310.5 \mathrm{IU} / \mathrm{L}$, AST predicted 1 month mortality with a sensitivity of $84.56 \%$ and specificity of $42.37 \%$ (ROC-AUC: 0.665 , 95\% CI: 0.587 to $0.743, P<0.001$ ) and 2 year mortality with a sensitivity of $84.75 \%$ and specificity of $31.97 \%$ (ROC-AUC: $0.577,95 \% \mathrm{CI}: 0.520$ to $0.634, P=0.004$ ).

The 2 year all-cause mortality was significantly high along with an increasing level of ALT and AST (Table 4). Kaplan-Meier curves were constructed to explore the additive prognostic value of combinations of ALT and AST levels. We divided the study patients into four groups by $\geq 95$ th percentage of ALT and AST or not. 
Table 3 Association between liver enzymes and covariables in univariate analyses

\begin{tabular}{|c|c|c|c|c|}
\hline Variable & CC for ALT (SE) & $P$ value & CC for AST (SE) & $P$ value \\
\hline Age (year) & $30.456(3.275)$ & 0.002 & $-0.467(0.546)$ & 0.392 \\
\hline Male, n (\%) & $4.316(5.199)$ & 0.407 & $14.206(13.344)$ & 0.287 \\
\hline Living in the city & $-5.163(4.548)$ & 0.256 & $-17.423(11.674)$ & 0.136 \\
\hline Hospital days & $0.164(0.602)$ & 0.785 & $-2.807(1.545)$ & $0 . .069$ \\
\hline Dyslipidemia (n) & $-9.094(4.604)$ & 0.048 & $-20.057(11.818)$ & 0.090 \\
\hline Diabetes mellitus (n) & $-3.187(5.399)$ & 0.555 & $-11.918(13.859)$ & 0.390 \\
\hline Hypertension (n) & $1.631(4.536)$ & 0.719 & 3.295 (11.642) & 0.777 \\
\hline Myocardial disease (n) & $-17.047(9.239)$ & 0.065 & $-38.681(23.715)$ & 0.103 \\
\hline Killip classification & $30.456(3.275)$ & $<0.001$ & $83.942(8.406)$ & $<0.001$ \\
\hline Infarct-related coronary artery & $8.766(4.176)$ & 0.036 & $27.303(10.718)$ & 0.011 \\
\hline cardiac troponin I (ng/mL) & $0.116(0.037)$ & 0.002 & $0.809(0.094)$ & $<0.001$ \\
\hline Pre-TMl flow & $-29.730(5.587)$ & $<0.001$ & $-69.304(14.339)$ & $<0.001$ \\
\hline Multivessel disease ( $\geq 2$ vessels) & $7.055(4.539)$ & 0.120 & $17.559(11.650)$ & 0.132 \\
\hline
\end{tabular}

The all-cause mortality at 1 month and 2 years were significantly increased in patients with both AST and ALT levels $\geq 95$ th percentage $(P<0.001)$ (Fig. 2$)$.

\section{Discussion}

Liver function tests usually comprise ALT, AST, ALP, GGT, other nonenzymatic proteins (e.g., albumin), and heme metabolites, such as bilirubin. Among these markers, AST and ALT are often elevated in patients with STEMI [17]. Serum ALT is predominantly found in the liver. AST is mainly derived from liver and, hence, also considered as a marker of liver function; however, a significant portion of AST is derived from the heart and other tissues. Nevertheless, it remains unclear whether ALT or AST provide any long- or short-term independent prognostic value in STEMI patients who underwent PCI and whether ALT and AST levels are associated with any other risk factors.

To our knowledge, this is the first prospective study evaluating the association between ALT and AST and short- and long-term all-cause mortality in STEMI patients who underwent PCI. We demonstrated in the Chinese Han ethnic group that AST and ALT levels on admission were significant correlated with Killip classification, pre-TIMI flow, infarct-related coronary artery, and cardiac troponin I. Serum transaminases $\geq 95$ th percentage were associated with a significantly increased incidence of short- and long-term all-cause mortality.

Recent studies investigating the relationship between ALT and AST with mortality have yielded conflicting results. A large national population-based cohort study conducted in the United States showed a lack of association between overall or cardiovascular-disease (CVD) mortality with ALT [18]. However, a similar study of a population-based cohort of Caucasian persons aged 55 years or older found AST and ALT are associated with all-cause mortality [19]. Furthermore, a recent meta-analysis study with aggregate data on over 9.24 million participants and 242,953 instances of all-cause mortality found a comparatively moderate association of AST with all-cause mortality and geographical variations in the association of ALT with all-cause mortality risk in
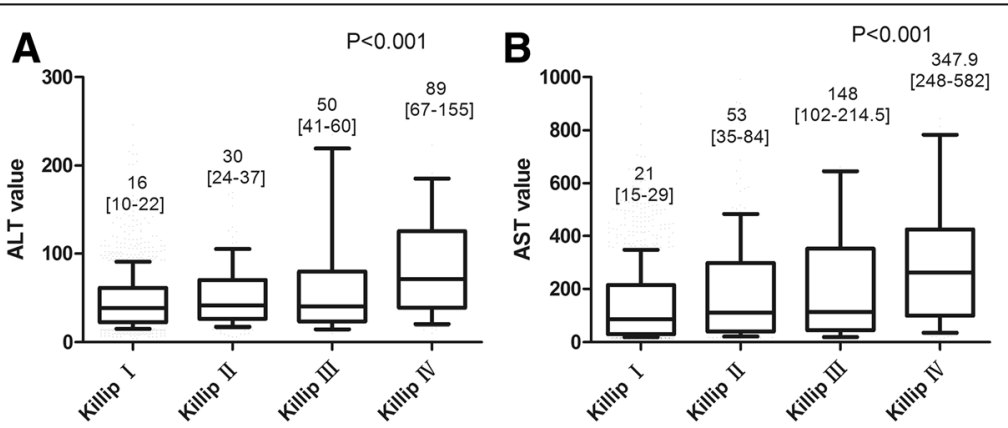

Fig. 1 ALT (a) and AST (b) values stratified according to Killip classification. The horizontal line shows the median value. The box showed the interquartile range. The vertical line shows the 10th-90th percentage 
Table 4 Association of liver enzymes with long-term all-cause mortality

\begin{tabular}{|c|c|c|c|c|c|c|c|c|c|c|}
\hline \multirow{3}{*}{ Category } & \multicolumn{10}{|c|}{ Association of ALT with all-cause mortality } \\
\hline & \multirow[t]{2}{*}{ ALT (unit/L) } & \multicolumn{3}{|c|}{ Crude univariate model } & \multicolumn{3}{|c|}{ Adjusted for age and sex } & \multicolumn{3}{|c|}{ Adjusted for all covariables } \\
\hline & & OR & $95 \% \mathrm{Cl}$ & $P$-value & OR & $95 \% \mathrm{Cl}$ & P-value & OR & $95 \% \mathrm{Cl}$ & $P$-value \\
\hline $\operatorname{ALT}(1)^{\mathrm{a}}$ & $\leq 23$ & 1 (ref) & & $<0.001$ & 1 (ref) & & $<0.001$ & 1 (ref) & & $<0.001$ \\
\hline $\operatorname{ALT}(2)$ & $>23$ to $\leq 39$ & 0.957 & $0.534-1.714$ & & 1.126 & $0.624-2.030$ & & 1.358 & $0.650-2.834$ & \\
\hline $\operatorname{ALT}(3)$ & $>39$ to $\leq 64$ & 0.914 & $0.506-1.648$ & & 1.124 & $0.618-2.041$ & & 1.413 & $0.658-3.036$ & \\
\hline $\operatorname{ALT}(4)$ & $>64$ to $\leq 125$ & 1.654 & $0.957-2.858$ & & 2.144 & $1.226-3.751$ & & 1.650 & $0.732-3.719$ & \\
\hline \multirow[t]{2}{*}{$\operatorname{ALT}(5)$} & $\geq 125$ & 5.370 & 2.899-9.948 & & 7.034 & $3.718-13.307$ & & 1.051 & $0.302-3.652$ & \\
\hline & \multicolumn{10}{|c|}{ Association of AST with all-cause mortality } \\
\hline \multirow[t]{2}{*}{ Category } & AST (unit/L) & \multicolumn{3}{|c|}{ Crude univariate model } & \multicolumn{3}{|c|}{ Adjusted for age and sex } & \multicolumn{3}{|c|}{ Adjusted for all covariables } \\
\hline & & OR & $95 \% \mathrm{Cl}$ & $P$-value & OR & $95 \% \mathrm{Cl}$ & $P$-value & OR & $95 \% \mathrm{Cl}$ & $P$-value \\
\hline AST $(1)^{\mathrm{a}}$ & $\leq 32$ & 1 (ref) & & $<0.001$ & 1 (ref) & & $<0.001$ & 1 (ref) & & $<0.001$ \\
\hline AST (2) & $>32$ to $\leq 93$ & 1.442 & $0.844-2.462$ & & 1.432 & $0.834-2.459$ & & 1.379 & $0.700-2.717$ & \\
\hline AST (3) & $>93$ to $\leq 233$ & 0.785 & $0.425-1.448$ & & 0.744 & $0.401-1.382$ & & 0.786 & $0.361-1.715$ & \\
\hline AST (4) & $>233$ to $\leq 492$ & 1.206 & $0.672-2.164$ & & 1.152 & $0.639-2.080$ & & 0.989 & $0.439-2.225$ & \\
\hline AST (5) & $\geq 492$ & 5.370 & 2.899-9.948 & & 5.699 & $3.030-10.718$ & & 1.796 & $0.588-5.481$ & \\
\hline
\end{tabular}

$H R$ hazard ratio, $\mathrm{Cl}$ confidence interval, $A L T$ alanine aminotransferase, $A S T$ aspartate aminotransferase

${ }^{a}$ Percentiles are shown in categories: (1) $\leq 25$ th percentage; (2) 25 th $-<50$ th percentage; (3) 50 th- $<75$ th percentage; (4) 75 th- $<95$ th percentage; (5) $\geq 95$ th percentage

general populations [7]. Interestingly, other studies have found elevation of common markers, including ALT and AST, in patients with heart failure and CVD with liver injury resulting from ischemia or congestion [20-23]. These results further confirm the association between ALT/AST and mortality risk. Considering that liver enzymes are routine parameters of liver function assessed in STEMI patients before PCI, we investigated the association between ALT/AST and all-cause mortality in the Chinese Han ethnic group.

Notably, increased ALT levels are found in STEMI patients with acute liver injury [24-26]. Cardiac disorders

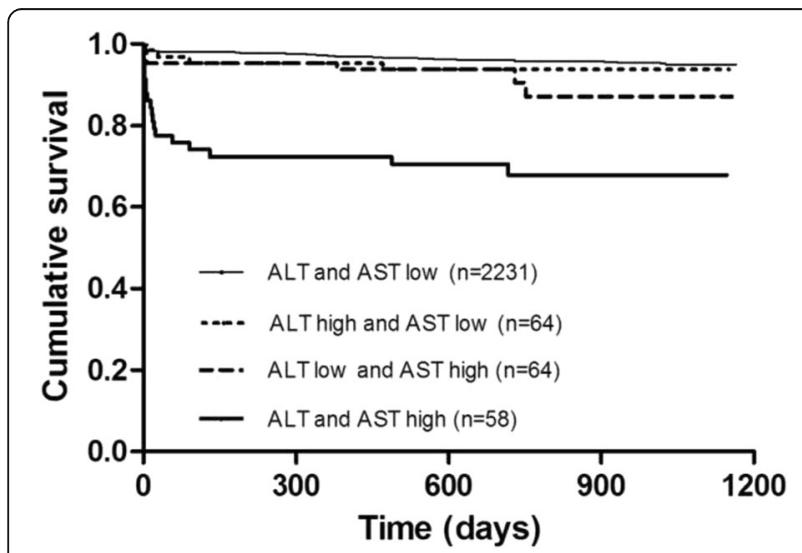

Fig. 2 Kaplan-Meier analysis. All-cause mortality according to combinations generated by having low or high values for ALT and AST. ALT and AST were defined low if under 95th percentage, high if above 95th percentage. ALT 95th percentage value $=125$ unit/L, AST 95th percentage value $=492$ unit/L contribute to the liver injury $[2,4,27,28]$. We excluded patients with chronic hepatitis to exclude liver injury derived from hepatic disorders. The infarct-related artery only strategy for patients with multivessel arteries disease presenting with STEMI in our center minimize potential impact of different strategies of complete vs. culprit-only revascularization [29]. The primary infarctrelated coronary artery in patients with ALT $\geq 95$ th percentage was LAD. Our findings support LAD occlusion as the factor most closely associated with left ventricular ejection fraction and with measures of left ventricular regional hypofunction, especially caused by proximal LAD [30, 31].

The liver has high metabolic activity and perfusion rate, and acute circulatory changes, such as cardiogenic shock resulting from an acute myocardial infarction, directly influence hepatic blood flow [32-34]. Every $10 \mathrm{mmHg}$ drop in arterial pressure decreases the hepatic blood flow by approximately $10 \%[33,35]$. Circulatory failure triggers compensatory mechanisms in the liver by increasing oxygen extraction from the blood up to $95 \%$, which results in hepatocellular dysfunction and elevation of AST/ALT [36]. The assessment for the presence and severity of circulatory failure were assessed by the Killip classification through physical examination, which showed the serum level of ALT and AST were high along with the increasing of the grade of Killip classification. Because hypoxic injury to the liver is a reversible subclinical condition accounting for over 50\% of dramatic serum aminotransferase activity identified in hospital admissions [37], our results demonstrating the 
predictive value of ALT and AST for short-term, but not long-term, all-cause mortality were not surprising. The influences of genetics on the ethnic and environmental factors, the dosage of prescription drugs, adherence to therapy and cardiac rehabilitation all had important roles on the long-term overall cardiovascular risk. Unless for ALT and AST levels $\geq 95$ th percentage, the all-cause mortality at 1 month and 2 years post-procedure both significantly increased.

\section{Study limitations}

This study had several limitations. This was an observational study based on data from a single center and the population belonging to a single ethnic group which located in an agricultural province of Northeast China with $52 \%$ of participants living in the countryside. The crude death rate of coronary heart disease is significantly different form urban citizens and rural residents in China [38]. The proportion of rural population may influence the result and the data may not reflect the general population of STEMI patients; however, compared to a multicenter study, this study design could be an advantage. The patients' data were imputed electronically by a relatively constant group of attending physicians. The severity and location of coronary lesions were based on visual assessments by the same operators. Ticagrelor was not available during the study period in our center which avoided different P2Y12 inhibitors affecting AST and ALT levels. The overall strategic management of patients, including PCI technique and device used during the procedure, were more homogeneous than would be in a multi-centered study. Second, we performed only a single measurement of ALT and AST for most patients admitted to our center. We tried to exclude patients with conditions potentially associated with liver cell damage, and we also excluded $5 \%$ of patients who did not have complete evaluation of liver enzymes at admission. Those limitations are balanced by our continuous admission and, in particular, by the avoidance of ascertainment bias that occurs in clinical studies of selected patients. Third, the results of our multivariate analysis may be biased due to the potential impact of important factors that are not available in our database. Fourth, validation of the cause of death was reported only in a portion of our cohort. Due to culture and social reasons, some patients' families did not know the exact cause of death.

\section{Conclusions}

In summary, to our knowledge, this is the first study to investigate the value of serum transaminases in patients with STEMI treated with primary PCI. Elevated transaminases significant correlated with Killip classification, preTIMI flow, infarct-related coronary artery, and cardiac troponin I. Moreover, the all-cause mortality at short- and long-term post-procedure was significantly increased in patients with levels of AST and ALT $\geq 95$ th percentage.

\section{Additional file}

Additional file 1: Three additional tables. Table S1. Predictors of major events at 1 month. Table S2. Predictors of major events at 24 month. Table S3. Percentile values of serum ALT and AST levels for men and women in the study population. (DOC $104 \mathrm{~kb}$ )

\section{Abbreviations}

ALP: Alkaline phosphatase; ALT: Alanine transaminase; AST: Aspartate aminotransferase; CKMB: Creatine kinase MB; GGT: $\gamma$-glutamyl transpeptidase; HDL-C: High-density lipoprotein cholesterol; LAD: Left anterior descending artery; LCX: Left circumflex coronary artery; LDL-C: Low-density lipoprotein cholesterol; NT-proBNP: N-terminal pro-brain natriuretic peptide; PCl: Percutaneous coronary intervention; RCA: Right coronary artery; ROC-AUC: Receiver operating characteristic area under the curve; STEMI: ST-segment elevation myocardial infarction; TC: Total cholesterol; TG: Triglyceride; TIMl: Thrombolysis-inmyocardial-infarction; Tn I: Cardiac troponin I

\section{Acknowledgements}

We thank Rose Mikulski who performed English language revision.

\section{Funding}

This work was supported by the funds from National Natural Science Foundation of China (81573230).

\section{Availability of data and materials}

The datasets used and analyzed during the current study are available from the corresponding author on request.

\section{Authors' contributions}

LQ and MG made main contribution to design of the study, drafted the manuscript, and performed the statistical analysis and participated. YC and YZ performed data collection and helped to draft the manuscript and make the statistical analysis. WHZ and LW participated in the design and coordination of the study and participated in the data collection. All authors have read and approved the final manuscript.

\section{Competing interests}

The authors declare that they have no competing interests.

Consent for publication

Not applicable.

Ethics approval and consent to participate

The study protocol was approved by the ethics review board of the First Hospital of Jilin University (No.2016-263). All patients gave written informed consent.

\section{Author details}

${ }^{1}$ The Cardiovascular Center, the First Hospital of Jilin University, 71 Xinmin Street, Changchun 130021, China. ${ }^{2}$ Laboratory for Cardiovascular Diseases, Institute of Translational Medicine, the First Hospital of Jilin University, Changchun, China. ${ }^{3}$ Key Laboratory for Cardiovascular Mechanism of Traditional Chinese Medicine, the First Hospital of Jilin University, Changchun, China.

Received: 7 October 2016 Accepted: 24 January 2017 Published online: 28 January 2017

\section{References}

1. Poelzl G, Ess M, Mussner-Seeber C, et al. Liver dysfunction in chronic heart failure: prevalence, characteristics and prognostic significance. Eur J Clin Invest. 2012:42:153-63.

2. Batin $P$, Wickens $M$, McEntegart $D$, et al. The importance of abnormalities of liver function tests in predicting mortality in chronic heart failure. Eur Heart J. 1995;16:1613-8. 
3. Allen LA, Felker GM, Pocock S, et al. Liver function abnormalities and outcome in patients with chronic heart failure: data from the Candesartan in Heart Failure: Assessment of Reduction in Mortality and Morbidity (CHARM) program. Eur J Heart Fail. 2009:11:170-7.

4. Alvarez AM, Mukherjee D. Liver abnormalities in cardiac diseases and heart failure. Int J Angiol. 2011;20:135-42

5. Fouad YM, Yehia R. Hepato-cardiac disorders. World J Hepatol. 2014;6:41-54.

6. Yun KE, Shin CY, Yoon YS, et al. Elevated alanine aminotransferase levels predict mortality from cardiovascular disease and diabetes in Koreans. Atherosclerosis. 2009:205:533-7.

7. Kunutsor SK, Apekey TA, Seddoh D, et al. Liver enzymes and risk of all-cause mortality in general populations: a systematic review and meta-analysis. Int J Epidemiol. 2014;43:187-201.

8. Lee TH, Kim WR, Benson JT, et al. Serum aminotransferase activity and mortality risk in a United States community. Hepatology. 2008;47:880-7.

9. Prati D, Taioli E, Zanella A, et al. Updated definitions of healthy ranges for serum alanine aminotransferase levels. Ann Intern Med. 2002;137:1-10.

10. Lee JK, Shim JH, Lee HC, et al. Estimation of the healthy upper limits for serum alanine aminotransferase in Asian populations with normal liver histology. Hepatology. 2010;51:1577-83.

11. Al-hamoudi W, Ali S, Hegab B, et al. Revising the upper limit of normal for levels of serum alanine aminotransferase in a Middle Eastern population with normal liver histology. Dig Dis Sci. 2013;58:2369-75.

12. Hochholzer W, Neumann FJ. The new 2015 ESC Guidelines for the management of acute coronary syndromes in patients presenting without persistent ST-segment elevation. Dtsch Med Wochenschr. 2016;141:782-5.

13. Killip 3rd T, Kimball JT. Treatment of myocardial infarction in a coronary care unit. A two year experience with 250 patients. Am J Cardiol. 1967;20:457-64.

14. Thygesen K, Alpert JS, Jaffe AS, et al. Third universal definition of myocardial infarction. Eur Heart J. 2012;33:2551-67.

15. Steg PG, James SK, Atar D, et al. ESC Guidelines for the management of acute myocardial infarction in patients presenting with ST-segment elevation. Eur Heart J. 2012;33:2569-619.

16. O'Gara PT, Kushner FG, Ascheim DD, et al. 2013 ACCF/AHA guideline for the management of ST-elevation myocardial infarction: a report of the American College of Cardiology Foundation/American Heart Association Task Force on Practice Guidelines. Circulation. 2013;127:e362-425.

17. Moon J, Kang W, Oh PC, et al. Serum transaminase determined in the emergency room predicts outcomes in patients with acute ST-segment elevation myocardial infarction who undergo primary percutaneous coronary intervention. Int J Cardiol. 2014;177:442-7.

18. Ruhl CE, Everhart JE. Elevated serum alanine aminotransferase and gamma-glutamyltransferase and mortality in the United States population. Gastroenterology. 2009;136:477-485.e411.

19. Koehler EM, Sanna D, Hansen BE, et al. Serum liver enzymes are associated with all-cause mortality in an elderly population. Liver Int. 2014;34:296-304.

20. Masoudkabir F, Karbalai S, Vasheghani-Farahani A, et al. The association of liver transaminase activity with presence and severity of premature coronary artery disease. Angiology. 2011;62:614-9.

21. Cagli K, Basar FN, Tok D, et al. How to interpret liver function tests in heart failure patients? Turk J Gastroenterol. 2015;26:197-203.

22. Moller S, Bernardi M. Interactions of the heart and the liver. Eur Heart J. 2013:34:2804-11.

23. Kavoliuniene A, Vaitiekiene A, Cesnaite G. Congestive hepatopathy and hypoxic hepatitis in heart failure: a cardiologist's point of view. Int J Cardiol. 2013;166:554-8

24. Ladue JS, Wroblewski F. The significance of the serum glutamic oxalacetic transaminase activity following acute myocardial infarction. Circulation. 1955;11:871-7

25. Tapper EB, Sengupta N, Bonder A. The incidence and outcomes of ischemic hepatitis: a systematic review with meta-analysis. Am J Med. 2015;128:1314-21.

26. Saner $\mathrm{FH}$, Heuer $\mathrm{M}$, Meyer $\mathrm{M}$, et al. When the heart kills the liver: acute liver failure in congestive heart failure. Eur J Med Res. 2009;14:541-6.

27. Samsky MD, Patel CB, DeWald TA, et al. Cardiohepatic interactions in heart failure: an overview and clinical implications. J Am Coll Cardiol. 2013;61:2397-405.

28. van Deursen VM, Damman $\mathrm{K}$, Hillege $\mathrm{HL}$, et al. Abnormal liver function in relation to hemodynamic profile in heart failure patients. J Card Fail. 2010;16:84-90.

29. Moretti C, D'Ascenzo F, Quadri G, et al. Management of multivessel coronary disease in STEMI patients: a systematic review and meta-analysis. Int J Cardiol. 2015;179:552-7.
30. Stadius ML, Maynard C, Fritz JK, et al. Coronary anatomy and left ventricular function in the first 12 hours of acute myocardial infarction: the Western Washington Randomized Intracoronary Streptokinase Trial. Circulation. 1985:72:292-301.

31. Brener SJ, Witzenbichler B, Maehara A, et al. Infarct size and mortality in patients with proximal versus mid left anterior descending artery occlusion: the Intracoronary Abciximab and Aspiration Thrombectomy in Patients With Large Anterior Myocardial Infarction (INFUSE-AMI) trial. Am Heart J. 2013:166:64-70.

32. Nikolaou M, Parissis J, Yilmaz MB, et al. Liver function abnormalities, clinical profile, and outcome in acute decompensated heart failure. Eur Heart J. 2013;34:742-9.

33. Naschitz JE, Slobodin G, Lewis RJ, et al. Heart diseases affecting the liver and liver diseases affecting the heart. Am Heart J. 2000;140:111-20.

34. Denis C, De Kerquennec C, Bernuau J, et al. Acute hypoxic hepatitis ('liver shock'): still a frequently overlooked cardiological diagnosis. Eur J Heart Fail. 2004;6:561-5.

35. Jolobe OM. Jaundice as a presentation of heart failure. J R Soc Med. 2005:98:532.

36. Eipel C, Abshagen K, Vollmar B. Regulation of hepatic blood flow: the hepatic arterial buffer response revisited. World J Gastroenterol. 2010:16:6046-57.

37. Emmanuel A, Inns S. Lecture notes in gastroenterology and hepatology. 1st ed. Blackwell Pub; 2011.

38. Hu SS, Kong LZ, Gao RL, et al. Outline of the report on cardiovascular disease in China, 2010. Biomed Environ Sci. 2012;25:251-6.

\section{Submit your next manuscript to BioMed Central and we will help you at every step:}

- We accept pre-submission inquiries

- Our selector tool helps you to find the most relevant journal

- We provide round the clock customer support

- Convenient online submission

- Thorough peer review

- Inclusion in PubMed and all major indexing services

- Maximum visibility for your research

Submit your manuscript at www.biomedcentral.com/submit 